\title{
The FAST Project - A Next Generation UHECR Observatory
}

\author{
Toshihiro Fujii ${ }^{1, a}$, Max Malacari², Jose A. Bellido ${ }^{3}$, Pavel Horvath ${ }^{4}$, Miroslav Hrabovsky ${ }^{4}$, Jiaqi \\ Jiang $^{2}$, Dusan Mandat ${ }^{5}$, Ariel Matalon ${ }^{2}$, John N. Matthews ${ }^{6}$, Pavel Motloch ${ }^{2}$, Miroslav Palatka ${ }^{5}$, \\ Miroslav Pech ${ }^{5}$, Paolo Privitera ${ }^{2}$, Petr Schovanek ${ }^{5}$, Stan B. Thomas ${ }^{6}$, and Petr Travnicek ${ }^{5}$ \\ ${ }^{1}$ Institute for Cosmic Ray Research, University of Tokyo, Kashiwa, Chiba, Japan \\ ${ }^{2}$ Kavli Institute for Cosmological Physics, University of Chicago, Chicago, Illinois, USA \\ ${ }^{3}$ Department of Physics, University of Adelaide, Adelaide, S.A., Australia \\ ${ }^{4}$ Palacky University, RCPTM, Olomouc, Czech Republic \\ ${ }^{5}$ Institute of Physics of the Academy of Sciences of the Czech Republic, Prague, Czech Republic \\ ${ }^{6}$ High Energy Astrophysics Institute and Department of Physics and Astronomy, University of Utah, Salt Lake \\ City, Utah, USA
}

\begin{abstract}
We present a concept for large-area, low-cost detection of ultrahigh-energy cosmic rays (UHECRs) with a Fluorescence detector Array of Single-pixel Telescopes $(\text { FAST })^{\mathrm{b}}$, addressing the requirements for the next generation of UHECR experiments. In the FAST design, a large field of view is covered by a few pixels at the focal plane of a mirror or Fresnel lens. We report on the first results of a FAST prototype installed at the Telescope Array (TA) site, consisting of a single $200 \mathrm{~mm}$ photomultiplier tube (PMT) at the focal plane of a $1 \mathrm{~m}^{2}$ Fresnel lens system taken from the prototype of the JEM-EUSO experiment. We also report on the status of the full-scale FAST prototype soon to be installed at the TA site, comprising a segmented spherical mirror of $1.6 \mathrm{~m}$ diameter and a $2 \times 2$ PMT camera.
\end{abstract}

\section{The FAST Project}

The origin and nature of ultrahigh-energy cosmic rays (UHECRs) is one of the most intriguing mysteries in particle astrophysics [1]. Given their minute flux, less than one per century per square kilometre at the highest energies, a very large area must be instrumented to collect significant statistics. The energy, arrival direction, and mass composition of UHECRs can be inferred from studies of the cascade of secondary particles (Extensive Air Shower, EAS) produced by their interaction with the Earth's atmosphere. Two well-established techniques are used for UHECR detection: 1) arrays of detectors (e.g. plastic scintillators and water-Cherenkov stations) sample EAS particles reaching the ground; 2) large field of view telescopes allow for reconstruction of the shower development in the atmosphere by imaging ultraviolet (UV) fluorescence light from atmospheric nitrogen excited by EAS particles.

The Pierre Auger Observatory (Auger) [2] and the Telescope Array Experiment (TA) [3, 4], the two largest UHECR experiments currently in operation, combine the two techniques, with arrays of particle detectors overlooked by fluorescence detector (FD) telescopes. Auger covers an area of over

\footnotetext{
ae-mail: fujii@icrr.u-tokyo.ac.jp

$\mathrm{b}_{\text {webpage: }}$ www.fast-project.org
} 
$3000 \mathrm{~km}^{2}$ close to the town of Malargüe in the province of Mendoza, Argentina. TA is located near the town of Delta in central Utah, USA, and covers an area of $700 \mathrm{~km}^{2}$. Significant advances in our understanding of UHECRs have been achieved in the last decade by these experiments [5]. However, these results are limited by statistics at the highest energies. To further advance the field, the next generation of experiments will require an aperture which is larger by an order of magnitude. This may be accomplished by fluorescence detection of UHECR showers from space, as in the proposed JEM-EUSO mission [6], or with a giant ground array. Low-cost, easily-deployable detectors will be essential for a future ground-based experiment.

We present a ground-based FD telescope concept which would fulfill these requirements. The Fluorescence detector Array of Single-pixel Telescopes (FAST) would consist of compact FD telescopes featuring a smaller light-collecting area and far fewer pixels than current-generation FD designs, leading to a significant reduction in cost. In the FAST design, a $30^{\circ} \times 30^{\circ}$ field of view is covered by just a few $200 \mathrm{~mm}$ PMTs at the focal plane of a mirror or Fresnel lens of $A \sim 1 \mathrm{~m}^{2}$. FAST stations, powered by solar panels and with wireless connection, could be deployed in an array configuration to cover a very large area at low cost.

\section{A first test of the FAST concept at the Telescope Array site}
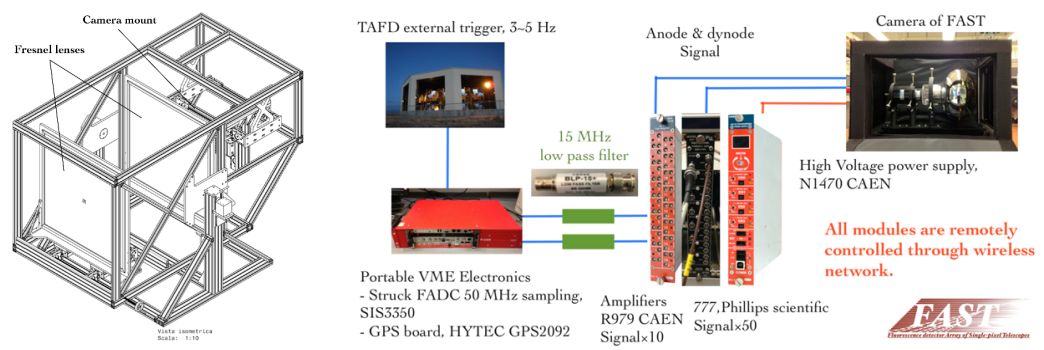

Figure 1. The EUSO-TA telescope optics (left) [7] and the DAQ system of the FAST prototype (right). The dimensions of the of the EUSO-TA optics are approximately $1.8 \mathrm{~m} \times 2.0 \mathrm{~m} \times 2.6 \mathrm{~m}(\mathrm{H} \times \mathrm{W} \times \mathrm{L})$. The FAST prototype camera, consisting of a single $200 \mathrm{~mm}$ PMT and a UV transparent filter, was installed at the focal plane of the telescope.

A first test of the FAST concept was performed profiting from the existing infrastructure of the JEM-EUSO experiment at the TA site in Utah, where a prototype [7] is currently installed for a comprehensive test of the optics and electronics of this space-based detector. The light collecting area $\left(\sim 1 \mathrm{~m}^{2}\right)$ and circular field of view $\left(\sim 7^{\circ}\right.$ radius) of the JEM-EUSO prototype telescope (EUSO-TA telescope) are close to the FAST reference design (for a single pixel), providing a perfect test-bed for the FAST concept. Its optical system consists of two $1 \mathrm{~m}^{2}$ Fresnel lenses, with a UV transparent acrylic plate placed at the diaphragm for protection (Figure 1). For the purpose of the FAST test we installed an 200 mm PMT (mod. R5912-03, Hamamatsu) and an AC-coupled active base (mod. E7694-01, Hamamatsu) at the focal plane of the telescope. A UV band-pass filter (mod. Schott MUG6 glass) was placed in front of the PMT to reduce the night sky background. The electronics and Data Acquisition System (DAQ) of the FAST prototype was built from commercial modules (Figure 1) [8]. Whenever any of the FD telescopes in the adjacent TA building were triggered by a candidate UHECR shower an external trigger was issued to the FAST DAQ, with a typical rate of $\sim 3 \mathrm{~Hz}$. 


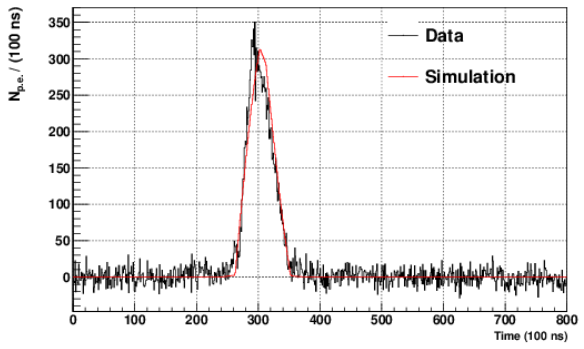

(a)

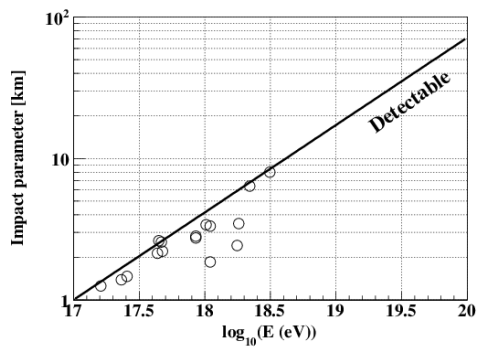

(b)

Figure 2. Measured signal and simulated expectation for a vertical UV laser (a). Correlation between the impact parameter and energy of the 16 cosmic ray shower candidates detected by FAST (b). Both shower parameters were obtained from the TA standard reconstruction. The line indicates the maximum detectable distance consistent with our limited data set [8].

UV laser shots are used for calibration of FD telescopes and atmospheric monitoring [9, 10]. While traveling up through the atmosphere, the laser light scatters on air molecules and aerosol particles into the FD field of view, producing signals similar to a UHECR shower. Figure 2.a shows the measured signal and simulated expectation from a UV vertical laser at a distance of $6 \mathrm{~km} \mathrm{[11].} \mathrm{The}$ energy of the laser pulse is $\sim 2.2 \mathrm{~mJ}$, approximately equivalent in intensity to $\mathrm{a} \approx 10^{19.2} \mathrm{eV}$ shower.

A shower signal search was performed, driven by well reconstructed TA FD events which generated an external trigger for the FAST DAQ. We first selected TA FD events with a reconstructed shower geometry passing through the field of view of the FAST prototype. We then searched the corresponding FAST FADC traces for pulses with a maximum signal greater than $5 \sigma$. As a result, we found 16 shower candidates in the 83 hour dataset, with an estimated background of $<1$ event. Although small, this sample provides an estimate of the sensitivity of the FAST prototype. The correlation between the impact parameter (i.e. the distance of closest approach of the shower axis with respect to the FAST prototype) and the energy of the 16 showers is plotted in Figure 2.b, with shower parameters given by the standard reconstruction of the TA FD [12].

\section{The Full-scale FAST prototype}

Motivated by these encouraging results using the EUSO-TA telescope optics, we have developed a full-scale FAST prototype as shown in Figure 3. This prototype consists of a segmented spherical mirror of $1.6 \mathrm{~m}$ diameter and a UV band-pass filter with a $1 \mathrm{~m}^{2}$ aperture. Four $200 \mathrm{~mm}$ PMTs and active bases are installed at the focal plane of the segmented mirror in a $2 \times 2$ matrix, covering a $25^{\circ} \times 25^{\circ}$ field of view. The telescope is covered with a shroud to shield the optical system from dust and stray light.

\section{Summary and Future Plans}

We have presented a novel concept for a next-generation air-shower fluorescence detector, which features just a few pixels covering a large field of view. We confirmed the validity of the FAST concept with test mesurements made using the exiting EUSO-TA telescope prototype optics and a $200 \mathrm{~mm}$ PMT. We successfully observed a vertical UV laser along with 16 UHECR showers in time 

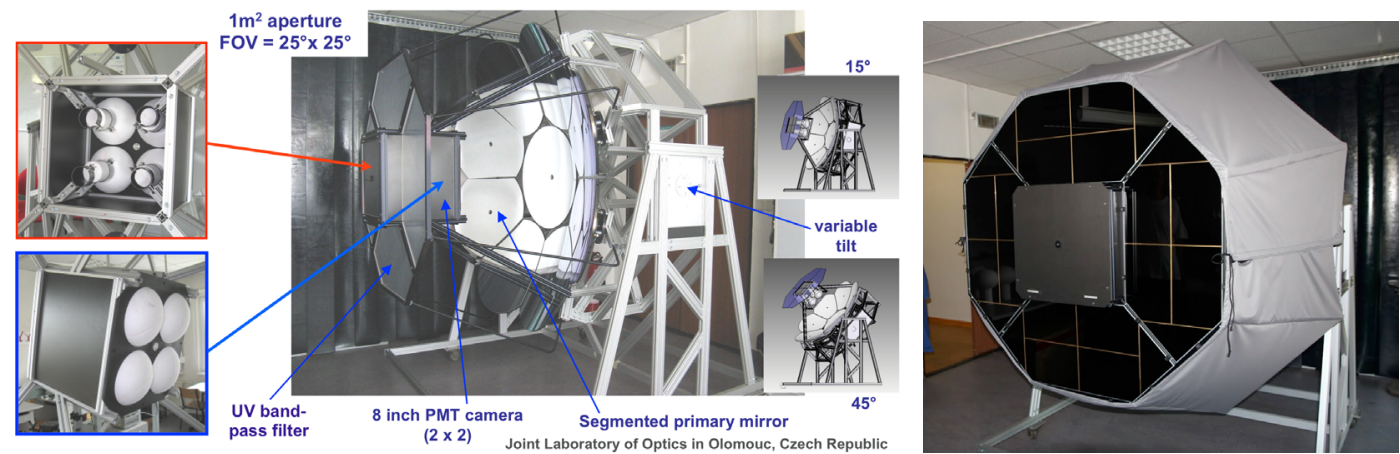

Figure 3. The full-scale FAST prototype being constructed at the Joint Laboratory of Optics in Olomouc, Czech Republic.

coincidence with the TA FD telescopes. Based on these encouraging results, we have developed a full-scale FAST prototype telescope for future installation at the Telescope Array site.

\section{Acknowledgements}

This work was supported in part by NSF grant PHY-1412261 and by the Kavli Institute for Cosmological Physics at the University of Chicago through grant NSF PHY-1125897 and an endowment from the Kavli Foundation and its founder Fred Kavli. The Czech authors gratefully acknowledge the support of the Ministry of Education, Youth and Sports of the Czech Republic project No. LG15014, LE13012, LO1305. This work was supported by the Japan Society for the Promotion of Science through the Grant-in-Aid for Young Scientist (A) 15H05443, Grant-in-Aid for JSPS Research Fellow 16J04564 and JSPS Fellowship H25-339, H28-4564.

\section{References}

[1] A.A. Watson, Rep.Prog.Phys. 77, 036901 (2014), 1310.0325

[2] A. Aab et al. (Pierre Auger Collaboration), Nucl. Instrum. Meth. A798, 172 (2015), 1502 . 01323

[3] H. Tokuno et al. (Telescope Array Collaboration), Nucl.Instrum.Meth. A676, 54 (2012), 1201.0002

[4] T. Abu-Zayyad et al. (Telescope Array Collaboration), Nucl.Instrum.Meth. A689, 87 (2012), 1201.4964

[5] K.H. Kampert, P. Tinyakov, C.R.Phys. 15, 318 (2014), 1405. 0575

[6] Y. Takahashi (JEM-EUSO Collaboration), New J.Phys. 11, 065009 (2009), 0910 . 4187

[7] Y. Takizawa et al. (JEM-EUSO Collaboration), Proc. of the 33rd International Cosmic Ray Conference, Rio de Janeiro, Brazil ID0832 (2013), 1307.7071

[8] T. Fujii et al., Astropart. Phys. 74, 64 (2016), 1504.00692

[9] B. Fick et al., J.Instrum. 1, P11003 (2006)

[10] S. Udo et al., Proc. of the 30th International Cosmic Ray Conference, Merida, Mexico 5, 1021 (2007)

[11] K. Yamazaki et al. (Telescope Array Collaboration), Proc. of the 33rd International Cosmic Ray Conference, Rio de Janeiro, Brazil ID0526 (2013)

[12] T. Fujii et al. (Telescope Array Collaboration), AIP Conf.Proc. 1367, 149 (2011) 